\title{
The two-hole ground state of the Hubbard-Anderson model, approximated by a variational RVB-type wave function
}

\author{
M.R.M.J. Traa, W.J. Caspers and E.J. Banning \\ Center for Theoretical Physics, University of Twente, P.O. Box 217, 7500 AE Enschede, \\ The Netherlands
}

Received 30 June 1993

In this paper the Hubbard-Anderson model on a square lattice with two holes is studied. The ground state (GS) is approximatcd by a variational RVB-type wave function. The holes interact by exchange of a localized spin excitation (SE), which is created or absorbed if a hole moves to a nearest-neighbour site. An SE can move over the sublattice on which it is created. A variational calculation of the GS and the GS-energy is performed for an open-ended $4 \times 4$ lattice with two holes with the restriction that the SE is neighbouring both holes and does not move over its sublattice. It is found that the two holes prefer a bound state in which their mutual distance is 1 or $\sqrt{2}$ (with lattice spacing 1 ).

\section{Introduction}

Since the discovery of high- $T_{\mathrm{c}}$ superconducting oxides there has been a growing interest in strongly correlated electron systems. Anderson [1] has suggested that the physics of these oxides is contained in the two-dimensional (2D), large- $U$, single-band Hubbard model. In this model doubly occupied sites are prohibited and the Hubbard Hamiltonian can be transformed into an effective Hamiltonian, called the Hubbard-Anderson (H-A) Hamiltonian [2],

$$
\begin{aligned}
& H_{\mathrm{H}-\mathrm{A}}=H_{1}+H_{2}+H_{3}, \\
& H_{1}=-t \sum_{\langle i, j\rangle} \sum_{\sigma}\left(c_{i \sigma}^{\dagger} c_{j \sigma}+\text { H.c. }\right), \\
& H_{2}=2 \frac{t^{2}}{U} \sum_{\langle i, j\rangle} \sum_{\sigma}\left(c_{i \sigma}^{\dagger} c_{i-\sigma} c_{j-\sigma}^{\dagger} c_{j \sigma}-n_{i \sigma} n_{j-\sigma}\right),
\end{aligned}
$$




$$
H_{3}=-\frac{t^{2}}{U} \sum_{\langle i, j, k\rangle} \sum_{\sigma}\left[\left(c_{i \sigma}^{\dagger} c_{j \sigma} c_{j-\sigma}^{\dagger} c_{k-\sigma}+c_{i \sigma}^{\dagger} n_{j-\sigma} c_{k \sigma}\right)+\text { H.c. }\right]
$$

We consider a positive hopping parameter $t$ and a large on-site Coulomb repulsion $U$, so that $0<t \leqslant U$. A pair of nearest-neighbour sites is denoted by $\langle i, j\rangle$, a triple for which $i$ and $k$ are different nearest neighbours of $j$ by $\langle i, j, k\rangle$. A site is either occupied by an electron with $z$-component of its $\operatorname{spin} \frac{1}{2} \sigma$, $\sigma= \pm 1(\hbar=1)$, or empty, i.e. occupied by a hole.

In this paper we study the two-hole ground state (GS) of the $\mathrm{H}-\mathrm{A}$ model on a $4 M \times 4 N$ square lattice with periodic boundary conditions. In section 2 the system without holes is considered. Its GS, the pseudo vacuum, is approximated by an RVB-type wave function. Two holes are made by an operator acting on the pseudo vacuum. In sections 3 and 4 the zeroth- and first-order GS of the two-hole system are described. A numerical study of the $4 \times 4$ openended system is discussed in section 5 .

\section{The system without holes}

At half filling, i.e. all sites occupied by one electron, the $\mathrm{H}-\mathrm{A}$ model is equivalent to a spin- $\frac{1}{2}$ antiferromagnetic (AF) Heisenberg model. Disregarding an additive constant, $H_{\mathrm{H}-\mathrm{A}}$ reduces to

$$
H_{\mathrm{Heis}}=J \sum_{\langle i, j\rangle} S_{i} \cdot S_{j}, \quad J=4 \frac{t^{2}}{U},
$$

with $\langle i, j\rangle$ denoting nearest-neighbour sites on the square lattice.

Liang, Doucot and Anderson proposed a variational RVB-type wave function for the GS of this model [3]. It is a singlet state and it obeys the sign rule for the GS of a spin- $\frac{1}{2}$ AF Heisenberg model on a bipartite lattice, derived by Marshall [4]. We rewrite the GS wave function (eq. 2 of [3]) in the form of a determinant with creation operators and denote it by $|0\rangle$, the pseudo vacuum. It is convenient to divide the square lattice into an odd and an even numbered sublattice. See fig. 1.

Then,

$$
|0\rangle=\left|\begin{array}{llll}
(12) & (14) & \cdots & (116 M N) \\
(32) & (34) & \cdots & (316 M N) \\
\vdots & & & \\
(16 M N-12) & (16 M N-14) & \cdots & (16 M N-116 M N)
\end{array}\right||\rangle
$$




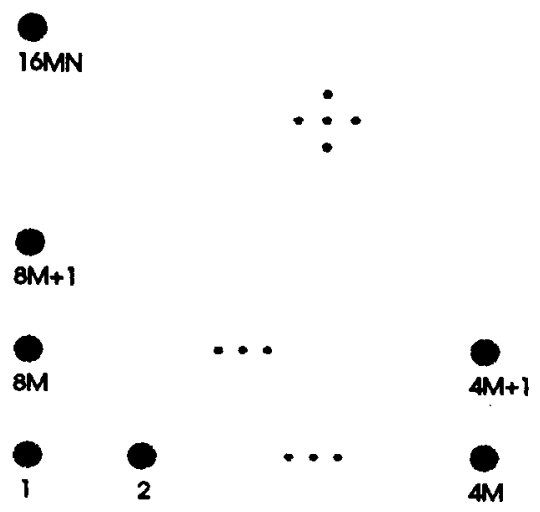

Fig. 1. Numbering of lattice, convenient for determinant notation.

The symbol $(i j)$ denotes the product of a positive parameter $h_{i j}$ and a combination of electron creation operators representing a singlet bond on sites $i$ and $j$, i.e.

$$
(i j)=h_{i j}\left(c_{i+}^{\dagger} c_{j-}^{\dagger}-c_{i-}^{\dagger} c_{j+}^{\dagger}\right) .
$$

Such a bond only connects sites of different sublattices. The parameter $h_{i j}$ can be interpreted as a weight factor for a bond as a function of its length. The real vacuum is denoted by |\rangle . Note that $(i j)$ and $(k l)$ commute for distinct $i, j, k$ and $l$, so that the determinant obeys the usual determinant calculation rules. As [3] mentions, the strong but tenable assumption is made that the amplitude of each bond covering in $|0\rangle$ can be factorized into a product of bond amplitudes. In [3] the values of the bond amplitudes $h_{i j}$ which lead to the lowest GS-energy of a $32 \times 32$ lattice are variationally determined. The tendency is that the bond amplitude $h_{i j}$ decays fast with increasing bond length.

\section{Zeroth-order states of the two-hole system}

In [5] the zeroth-order GS for the one-hole and two-hole system are discussed in terms of Ising configurations [6]. It turns out that in zeroth order the holes move over their quarter lattices (QL), i.e. they make steps over two lattice spacings in the $x$ and $y$ direction. With zeroth-order GS is meant the GS of the dominant part of $H_{\mathrm{H}-\Lambda}$, called $H_{0}$. This GS is characterized by the fact that it is non-frustrated [5]. The first-order GS is the GS of $H_{\mathrm{H}-\mathrm{A}}$, for which $H_{\mathrm{H}-\mathrm{A}}-H_{0}$ is used to add frustrated components to the zeroth-order GS in an optimal way. I.e. those frustrated components are added that couple to the 
zeroth-order components in the energetically most favourable way. The frustrated components form "bridges" between the zeroth-order components.

In this paper we study the GS of the two-hole system in the RVB language. Therefore, consider a creation operator for a singlet pair of holes on sites $i_{0}$ on the odd sublattice and $j_{0}$ on the even sublattice: $c_{j_{0}-} c_{i_{0}{ }^{+}}-c_{j_{0}+} c_{i_{0}-}$. Then

$$
\left(c_{j_{0}-} c_{i_{0}+}-c_{j_{0}+} c_{i_{0}-}\right)|0\rangle
$$

has the same phases for all Ising configurations and the same total spin $(S=0)$ as

$$
\begin{gathered}
\left|\left(i_{0} j_{0}\right)\right\rangle=(-1)^{\left(i_{0}+j_{0}-3\right) / 2} \\
\times\left|\begin{array}{llllll}
(12) & \cdots & \left(1 j_{0}-2\right) & \left(1 j_{0}+2\right) & \cdots & (116 M N) \\
\vdots & & & & & \\
\left(i_{0}-22\right) & \cdots & \left(i_{0}-2 j_{0}-2\right) & \left(i_{0}-2 j_{0}+2\right) & \cdots & \left(i_{0}-216 M N\right) \\
\left(i_{0}+22\right) & \cdots & \left(i_{0}+2 j_{0}-2\right) & \left(i_{0}+2 j_{0}+2\right) & \cdots & \left(i_{0}+216 M N\right) \\
\vdots & & & & & \\
(16 M N-12) & \cdots & \left(16 M N-1 j_{0}-2\right) & \left(16 M N-1 j_{0}+2\right) & \cdots & (16 M N-116 M N)
\end{array}\right||\rangle .
\end{gathered}
$$

The parameters $h_{i j}$ may differ from those in $|0\rangle$. They are expected to stay positive, because for positive $h_{i j}$ the spin background obeys Marshall's sign rule and therefore has optimal $\mathrm{H}_{2}$-energy. This corresponds to the experimental fact that the copperoxide-planes of high- $T_{\mathrm{c}}$ superconductors have an $\mathrm{AF}$ spin ordering.

Throughout sections 3 and 4 we concentrate on the correct treatment of the phase factors, which are +1 or -1 , because we deal with real states and expressions. Doing this, we keep in mind that all parameters are determined variationally when at last we minimize the energy-expectation value of the GS-approximation.

The zeroth-order GS is built up from two-hole configurations like in eq. (8). Its structure is given in [5] and can be found by splitting $H_{\mathrm{H}-\mathrm{A}}$ into

$$
H_{0}=H_{2}+H_{3,0} \quad \text { and } \quad H^{\prime}=H_{1}+H_{3,1} \text {. }
$$

The $H_{0}$-GS with $S=0$ is fourfold degenerate:

$$
|(1,2)\rangle,|(3,4)\rangle,|(1,4)\rangle \text { and }|(2,3)\rangle \text {. }
$$

The numbers indicate the quarter lattices (QL) on which the holes move around due to $H_{3,0}$. These $H_{0}$-GSs can be linearly combined into Bloch states [5]: 


$$
\begin{gathered}
|k\rangle=|(1,2)\rangle \pm|(3,4)\rangle, \quad k=\left\{\begin{array}{l}
(\pi, 0) \\
(\pi, \pi)
\end{array},\right. \\
|k\rangle=|(1,4)\rangle \pm|(2,3)\rangle, \quad k=\left\{\begin{array}{l}
(0, \pi) \\
(\pi, \pi)
\end{array} .\right.
\end{gathered}
$$

To give $|(1,2)\rangle$, etc., explicitly, we number the sites and the QL $(1-4)$ as in fig. 2. A site on QL $1,2,3,4$ has coordinates $(2 m, 2 n),(2 m-1,2 n),(2 m-1,2 n-$ $1),(2 m, 2 n-1)$. Then $|(1,2)\rangle$ reads

$$
\begin{aligned}
& |(1,2)\rangle=\sum_{m, n, p, q}(-1)^{m+n+p+q} \alpha_{m n p q} \\
& \quad\left(c_{2 p-1,2 q ;-} c_{2 m, 2 n ;+}-c_{2 p-1,2 q ;+} c_{2 m, 2 n ;-}\right)|0\rangle,
\end{aligned}
$$

in which the summation is over the integers $m, n, p$ and $q$, so that it corresponds to all combinations of site indices of QL 1 and 2. The phase factor is such that each $H_{3,0}$-matrix element is zero or negative for positive coefficients $\alpha_{m n p q}$ [5]. The coefficients depend on the distance between the holes and must be determined variationally. In the calculations we work with real coefficients to allow them to be rendered negative by the minimalization procedure. The expression $\left(c_{2 p-1,2 q ;-} c_{2 m, 2 n ;+}-c_{2 p-1,2 q ;+} c_{2 m, 2 n ;-}\right)|0\rangle$, shorthand $\left(c_{j_{0}-} c_{i_{0^{+}}}-c_{j_{0}+} c_{i_{0}-}\right)|0\rangle$, is (disregarding amplitudes) equal to $\left|\left(i_{0} j_{0}\right)\right\rangle$ in eq. (8). For the phase factor, it is convenient to number the sites as described before eq. (13). For the determinant expression, however, it is better to use the numbering corresponding with eq. (8). The other three $H_{0}$-GSs are described analogously.
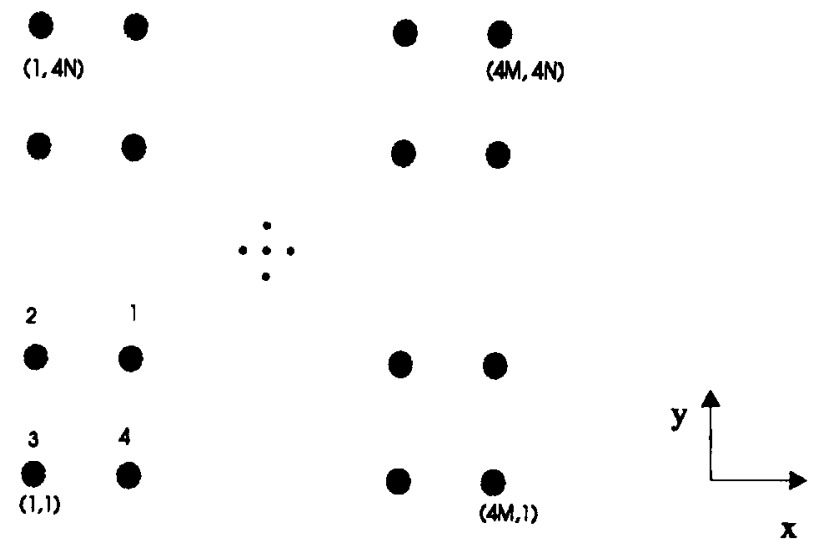

Fig. 2. Numbering of lattice as an $x y$-coordinate frame and numbering of $Q L$. 
Besides the states in eq. (10), one can construct a "bound" state. It corresponds to a different choice for $H_{0}$ :

$$
H_{0}=H_{2}+H_{3,1} \quad \text { and } \quad H^{\prime}=H_{1}+H_{3,0} .
$$

In the zeroth-order bound GS the holes are always nearest neighbours. Other positions of the holes lead to frustration if one considers the expectation value of the total $H_{\mathrm{H}-\mathrm{A}}$ (eq. (2.10) of [5]). Frustration is likely to raise the energy and therefore should be avoided, if possible. The bound GS is a Bloch state with $k=(0,0)$ and $S=0$. In zeroth order it reads (1-4 denote QL)

$$
|(0,0)\rangle=|(1,2)\rangle^{\prime}+|(3,4)\rangle^{\prime}+|(1,4)\rangle^{\prime}+|(2,3)\rangle^{\prime},
$$

with

$$
|(i, j)\rangle^{\prime}=\sum_{i_{0}, j_{0}}(-1)^{\mu}\left(c_{j_{0^{-}}} c_{i_{0^{+}}}-c_{j_{0}+} c_{i_{0^{-}}}\right)|0\rangle .
$$

The summation is over all sites $i_{0}$ of $\mathrm{Ql} i$ and $j_{0}$ of $\mathrm{QL} j$ with the restriction that $i_{0}$ and $j_{0}$ are nearest neighbours. The optimal $H_{3,1}$-phases are expressed by $(-1)^{\mu}$, with

$$
\mu= \begin{cases}0 & \text { for a pair of neighbour holes in the } x \text {-direction } \\ 1 & \text { for a pair of neighbour holes in the } y \text {-direction }\end{cases}
$$

The expression $\left(c_{j_{0}-} c_{i_{0}+}-c_{j_{0}+} c_{i_{0}-}\right)|0\rangle$ is (disregarding amplitudes) equal to $\left|\left(i_{0} j_{0}\right)\right\rangle$ in eq. (8).

\section{First-order states of the two-hole system}

The largest unfrustrated sets of two-hole configurations with $S=0$ are the zeroth-order states in eqs. (11), (12) and (15). We expand the sets by introducing the first-order two-hole configurations that result if $H_{1}$ acts on the zeroth-order configurations. The most important first-order term is $H_{1}$, because its amplitude $t$ is much larger than the amplitude of $H_{3}$, which is $t^{2} / U$. Both terms give an order-one contribution, because they act on a hole. This in contrast to $\mathrm{H}_{2}$, which is of the order of the number of electrons on the lattice.

The term $H_{1}$ displaces a hole to a neighbouring site, leaving behind a spin excitation (SE). Excitation, because the spin on that site has ferromagnetic phase relations with its neighbours. This is energetically unfavourable due to $\mathrm{H}_{2}$. The SE created by a hole can be absorbed by the same hole via the inverse 
$H_{1}$-process. But an SE can also give rise to a hole-hole interaction if it is absorbed by the second hole via $H_{1}$. If the second hole is not initially a neighbour of the first hole, the SE can move over its sublattice towards a neighbour site of the second hole via $\mathrm{H}_{2}$. Then it may be absorbed by the second hole via $H_{1}$. We found phase relations coupling the first-order configurations optimally to the zeroth-order configurations.

For example, consider the configuration in eq. (8) and let $j_{1}$, on the even sublattice, be a neighbour site of $i_{0}$. The configuration is depicted in fig. 3a for a $4 \times 4$ lattice. By one term of $H_{1}$ the hole on $i_{0}$ is displaced to $j_{1}$, leaving behind an SE on $i_{0}$ (fig. 3b). Disregarding amplitudes, one gets

$$
\begin{aligned}
& -\left(H_{1}\right)_{i_{0}, j_{1}}\left|\left(i_{0} j_{0}\right)\right\rangle=(-1)^{\left(i_{0}+j_{0}-3\right) / 2}
\end{aligned}
$$

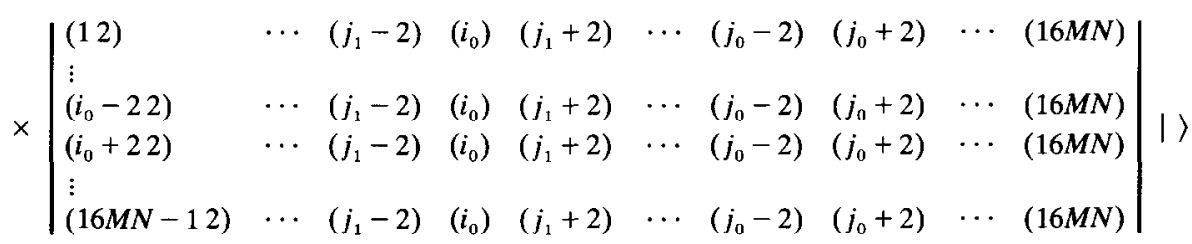

$$
\begin{aligned}
& =\left|\left(j_{1} j_{0}\right) ; i_{0}\right\rangle \text {. }
\end{aligned}
$$

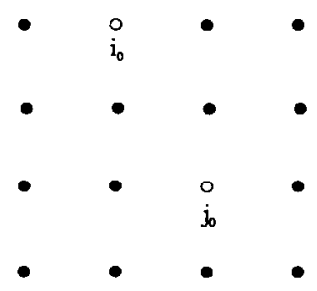

a) $\quad \mid\left(i_{b} j_{0}\right)>$

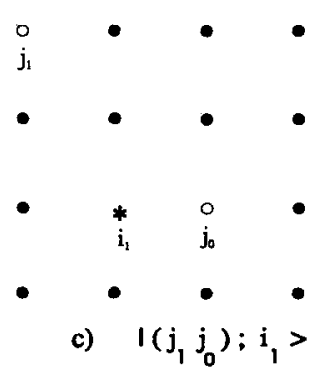

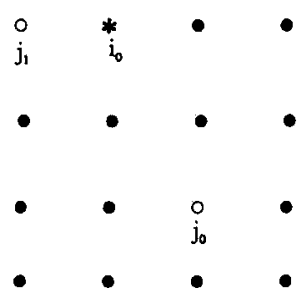

b) $\quad I\left(j_{1} j_{0}\right) ; i_{0}>$

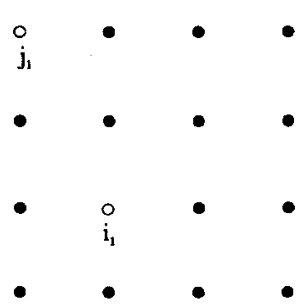

d) $\mid\left(i_{1} j_{1}\right)>$

Fig. 3. Interaction of two holes by exchange of an SE. 
Note that in eq. (17) the row labeled $i_{0}$ and the column labeled $j_{0}$ are gone. For convenience all columns after the first one are labeled with the second site of the singlet bond. The first site of the singlet bond is the row index. The column formerly labeled $j_{1}$ now has label $i_{0}$ : all its entries are singlets connecting sites of the odd sublattice to $i_{0}$, which is also on the odd sublattice.

We use $-H_{1}$ to derive a first-order configuration with an optimal connection to the zeroth-order configuration $\left|\left(i_{0} j_{0}\right)\right\rangle$, i.e.

$$
\left\langle\left(j_{1} j_{0}\right) ; i_{0}\left|\left(H_{1}\right)_{i_{0}, j_{1}}\right|\left(i_{0} j_{0}\right)\right\rangle<0 \text {. }
$$

One can show that the SE can move over its sublattice via $H_{2}$. Each position of the SE corresponds with a state of the same form as eq. (17) (figs. 3b and 3c). One can derive the following two rules:

Rule 1. If the SE, created by a hole, reaches a neighbour site of the other hole, it can be absorbed by that hole via $\mathrm{H}_{1}$ if and only if the final two-hole configuration has a minus sign relative to the initial two-hole configuration. The initial and final configuration belong to the zeroth-order GS.

For example, the initial configuration $\left|\left(i_{0} j_{0}\right)\right\rangle$ occurs in the GS as

$$
(-1)^{\varphi}\left|\left(i_{0} j_{0}\right)\right\rangle=(-1)^{\varphi}\left(c_{j_{0^{-}}} c_{i_{0^{+}}}-c_{j_{0^{+}}+} c_{i_{0}-}\right)|0\rangle,
$$

and the final configuration $\left|\left(i_{1} j_{1}\right)\right\rangle$ (fig. $3 \mathrm{~d}$ ) occurs in the GS as

$$
(-1)^{\varphi \pm 1}\left|\left(i_{1} j_{1}\right)\right\rangle=(-1)^{\varphi \pm 1}\left(c_{j_{1}-} c_{i_{1}+}-c_{j_{1}+} c_{i_{1}-}\right)|0\rangle
$$

The phases $(-1)^{\varphi}$ and $(-1)^{\varphi \pm 1}$ are determined by

- $H_{0}$; does one consider the GS-approximation with $(-1)^{m+n+p+q}$ or $(-1)^{\mu}$ ?

- which Bloch state one considers.

Rule 2. A hole can absorb the SE it created if and only if the final two-hole configuration has the same sign as the initial two-hole configuration. The initial and final configuration belong to the zeroth-order GS.

Both rules result in a reduction of the number of neighbour sites of a hole on which the SE can be absorbed. 


\section{Numerical study of the $4 \times 4$ open-ended system}

To see which state, the one based on $H_{2}+I_{3,0}$ or the one based on $\mathrm{H}_{2}+\mathrm{H}_{3,1}$, is energetically most favourable, we performed an exact variational calculation of the zeroth-order and first-order energies and states for the $4 \times 4$ open-ended system. Open-ended, to study the dependence of the energies and the coefficients $\alpha_{m n p q}$ (see eq. (13)) on the distance between the holes within a tractable size of the calculations.

Because of the system being open-ended one has to work with the states $|(1,2)\rangle$ etc. of eq. (10) and $|(1,2)\rangle^{\prime}$ etc. of eq. (15) instead of the Bloch states. The states are linearly combined in such a way that they transform according to irreducible representations of the point group of the square lattice, $\mathrm{C}_{4 \mathrm{v}}$ [7]. The coefficients $\alpha_{m n p q}$ now depend on the distance between the holes and the position of the hole pair on the $4 \times 4$ lattice. In eq. (15) one has to add a position dependent coefficient $\boldsymbol{\alpha}_{i_{0} j_{0}}$,

$$
|(i j)\rangle^{\prime}=\sum_{i_{0}, j_{0}}(-1)^{\mu} \alpha_{i_{0} j_{0}}\left(c_{j_{0}-} c_{i_{0}+}-c_{j_{0}+} c_{i_{0}-}\right)|0\rangle .
$$

Furthermore, in the first-order two-hole configurations the coefficients also depend on the position of the SE. The two-hole configurations that transform into one another by operators of $\mathrm{C}_{4 \mathrm{v}}$ have the same coefficient.

In the calculations we use the bond amplitudes $\left(h_{i j}\right.$ of eq. (6)) of the best GS-approximation of the pseudo vacuum of [3] and interpolated values for the amplitudes of singlet bonds of lengths 2,4 and 6 . The bond amplitudes are kept fixed. See table I. The coefficients $\alpha$ are the only variational parameters in our calculations.

First, consider the states of eq. (10). Define

$$
|(1,2)\rangle=e_{1}, \quad|(3,4)\rangle=e_{2}, \quad|(1,4)\rangle=e_{3} \quad \text { and } \quad|(2,3)\rangle=e_{4} .
$$

These four $\left(\mathrm{H}_{2}+\mathrm{H}_{3,0}\right)$-GSs are linearly combined into four orthogonal states, transforming according to irreducible representations of $\mathrm{C}_{4 \mathrm{v}}$ [7]:

Table I

Bond amplitudes of singlet bonds.

\begin{tabular}{ll}
\hline Manhattan length of singlet bond & Bond amplitude \\
\hline 1 & 1.00000 \\
2 & 0.30000 \\
3 & 0.12500 \\
4 & 0.07000 \\
5 & 0.03125 \\
6 & 0.01000 \\
\hline
\end{tabular}


$|1\rangle=e_{1}+e_{2}+e_{3}+e_{4}$ transforms according to the $1 \mathrm{D}$ irreducible representation $\mathrm{A}_{1}$.

$|2\rangle=e_{1}+e_{2}-e_{3}-e_{4}$ transforms according to the 1D irreducible representation $\mathrm{B}_{1}$.

$\left|K_{1}\right\rangle=e_{1}-e_{2}-e_{3}+e_{4}$ and $\left|K_{2}\right\rangle=e_{1}-e_{2}+e_{3}-e_{4}$ transform according to the $2 \mathrm{D}$ irreducible representation $\mathrm{E}$.

The number of zeroth-order configurations and first-order configurations with an SE - arc denoted in column two and three of table II. Both rules of section 4 were used to derive optimal first-order configurations. Forced by the size of the computational problem, we only considered first-order configurations with the holes on Manhattan distance two and a non-moving SE neighbouring both holes. For states $|1\rangle$ and $|2\rangle$ the first-order configurations are given in figs. 4 and 5. The configurations of fig. 4 are only optimal on the $2 \times 2$ squares, hereafter denoted by $\ominus$. Optimal means that the coupling of the first-order configurations (always with an SE) to the zeroth-order configurations (always without an SE) obeys eq. (18). So, in an $\ominus$ each configuration of fig. 4 is optimally coupled with two zeroth-order configurations. On the $2 \times 2$

Table II

Results of the $4 \times 4$ open-ended system

\begin{tabular}{lllllll}
\hline$t / U=0.10$ & $\begin{array}{l}\text { \#0th order } \\
\text { confs. }\end{array}$ & $\begin{array}{l}\text { \#SE } \\
\text { confs. }\end{array}$ & $\left\langle H_{2}+H_{3,0}\right\rangle$ & $\left\langle H_{2}+H_{3,1}\right\rangle$ & $\left\langle H_{2}+H_{3}\right\rangle$ & $\left\langle H_{\mathrm{H}-\mathrm{A}}\right\rangle$ \\
\hline$|1\rangle$ & 64 & 32 & 5.43 & - & $-5.35^{*}$ & -7.42 \\
$|2\rangle$ & 64 & 36 & -5.43 & $-5.77^{* * *}$ & -5.60 & -7.85 \\
$\left|K_{1}\right\rangle$ & 64 & 34 & -5.43 & - & -5.43 & $-6.89^{*}$ \\
$\left|K_{2}\right\rangle$ & 64 & 34 & -5.43 & - & -5.43 & $-6.89^{*}$ \\
$|(0,0)\rangle$ & 24 & 36 & - & $-5.37^{* *}$ & $-5.37^{* *}$ & $-8.17^{* *}$ \\
\hline
\end{tabular}

$\begin{array}{cccccccccc}0 & * & & 0 & 0 & * & 0 & 0 & 0 \\ 0 & 0 & * & 0 & 0 & 0 & 0 & *\end{array}$

Fig. 4. "Diagonal" first-order configurations.

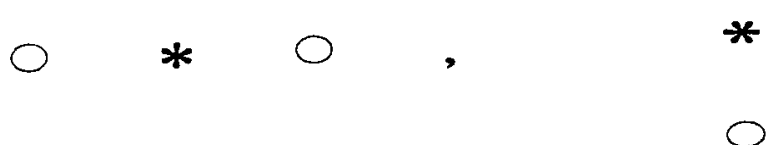

Fig. 5. "Stretched" first-order configurations. 


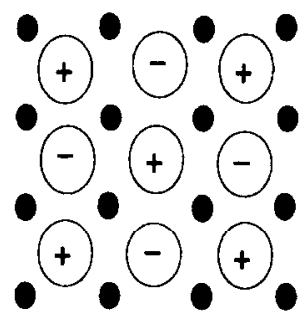

a)

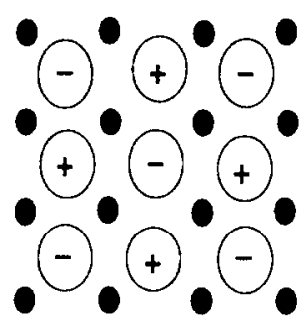

b)

Fig. 6. Pattern of optimal and non-optimal squares for (a) state $|1\rangle$ and (b) state $|2\rangle$.

squares, hereafter denoted by $\oplus$, the couplings are not optimal and we do not add any of the corresponding diagonal first-order configurations to the linear combination that approximates the GS. Fig. 6a shows the pattern of optimal squares $(\ominus)$ and non-optimal squares $(\oplus)$ for state $|1\rangle$. Fig. $6 \mathrm{~b}$ does the same for $|2\rangle$.

The configurations of fig. 5 are optimal on the whole $4 \times 4$ lattice and therefore all of them are added. For $\left|K_{1}\right\rangle$ the first-order configurations are given in fig. 7 . They are optimal on the whole $4 \times 4$ lattice. Analogously for $\left|K_{2}\right\rangle$ : see fig. 8 .

Now, consider the bound state. It transforms according to $B_{1}$ of $C_{4 v}$. We keep calling the bound state $|(0,0)\rangle$, although it is not a Bloch state anymore. The first-order configurations are of the type depicted in fig. 4 . They are optimal on the whole $4 \times 4$ lattice.

Table II gives the results of the variational calculations for the commonly used values $t=1$ and $U=10$.

- The entry $\left\langle j\left|H_{i}\right| j\right\rangle$ of table II means that $\left\langle j\left|H_{i}\right| j\right\rangle /\langle j \mid j\rangle$ is minimalized with respect to the coefficients $\alpha$. We will denote this minimalized energy by $\left\langle j\left|H_{i}\right| j\right\rangle$. The number of the coefficients $\alpha$ ranges from 4 to 18 .

- In columns four, five and six we worked with a linear combination only

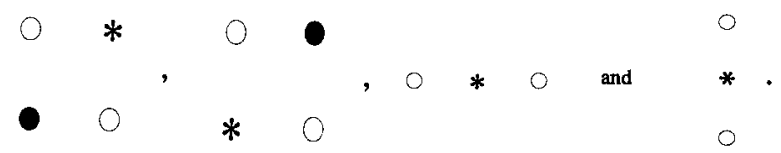

Fig. 7. First-order configurations of $\left|K_{1}\right\rangle$.

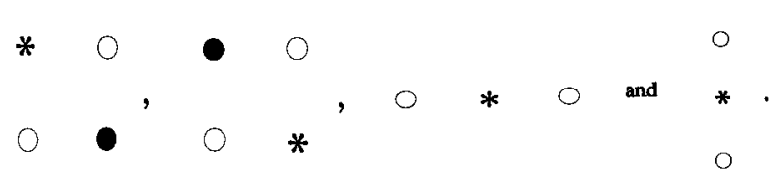

Fig. 8. First-order configurations of $\left|K_{2}\right\rangle$. 
containing the appropriate zeroth-order two-hole configurations. In column seven the appropriate first-order configurations were included as well.

- With $*$ is meant that the optimal $H_{3,0}$-phases, which are taken as starting point, are unstable. I.e. the minimilization of the energy renders some of the coefficients $\alpha$ negative, whereas the optimal $H_{3,0}$-phases correspond with positive $\alpha$-values.

- With $* *$ is meant that the optimal $H_{3,1}$-phases, starting point for the bound state, are stable.

- With $* * *$ is meant that the coefficients $\alpha$ are rendered with such values that all configurations have optimal $H_{3,1}$-phases.

- The other energies correspond to optimal $H_{3,0}$-phases.

\subsection{Comments on the results}

\subsubsection{Zeroth-order states}

- The energy $\left\langle H_{2}+H_{3,0}\right\rangle$ is the same for $|1\rangle-\left|K_{2}\right\rangle$, because they are linear combinations of $\left\{e_{i}, i=1-4\right\}$, which is the fourfold degenerate $\left(\mathrm{H}_{2}+\mathrm{H}_{3,0}\right)$-GS.

- The equality $\left\langle(0,0)\left|H_{2}\right| H_{3,1} \mid(0,0)\right\rangle=\left\langle\left\langle(0,0)\left|H_{2}+H_{3}\right|(0,0)\right\rangle\right.$ holds, because all $H_{3,0}$-matrix elements for the zeroth-order state $|(0,0)\rangle$ are zero.

- The states $\left|K_{1}\right\rangle$ and $\left|K_{2}\right\rangle$ transform according to the 2D irreducible representation $\mathrm{E}$ of $\mathrm{C}_{4 \mathrm{v}}$ and are orthogonal. All their energy-expectation values are exactly the same (in zeroth and first order), because $\mathrm{C}_{4 \mathrm{v}}$ is also the symmetry group of $H_{1}, H_{2}, H_{3,0}$ and $H_{3,1}$, separately.

- For the zeroth-order states we have

$$
\left\langle\left\langle 2\left|H_{2}+H_{3,1}\right| 2\right\rangle\right\rangle\left\langle\langle j | H _ { 2 } + H _ { 3 , 0 } | j \rangle \left\langle\left\langle\left\langle(0,0)\left|H_{2}+H_{3,1}\right|(0,0)\right\rangle,\right.\right.\right.
$$

with $j$ one of the set $\left\{1,2, K_{1}, K_{2}\right\}$.

Note that $|2\rangle$ in $\left\langle\left\langle 2\left|H_{2}+H_{3,1}\right| 2\right\rangle\right\rangle$ is an extension of $|(0,0)\rangle$ in $\langle(0,0)| H_{2}+$ $H_{3,1}|(0,0)\rangle$, because in $|2\rangle$ the holes can also be at Manhattan distance three or five.

- The line with label $|2\rangle$ shows that for the zeroth-order state $|2\rangle$ frustration in an elementary triangle results in a preference of $H_{3,0}$-phases above $H_{3,1^{-}}$ phases: in $\left\langle 2\left|H_{2}+H_{3}\right| 2\right\rangle$ all two-hole configurations have optimal $H_{3,0}$-phases. The underlying idea is explained in section 2 of [5]. There it is shown that $H_{3,1}$-phases lead to frustration in first order, whereas $H_{3,0}$-phases lead to frustration in second order.

The energy $\left\langle\left\langle 2\left|H_{2}+H_{3}\right| 2\right\rangle\right.$ is lower than $\left\langle(0,0)\left|H_{2}+H_{3}\right|(0,0)\right\rangle$. So, if one only considers $\mathrm{H}_{2}+\mathrm{H}_{3}$, there is a preference for $\mathrm{H}_{3,0}$-phases.

There is an additional cause for the system to prefer $H_{3,0}$-phases above $H_{3,1}$-phases as shown by $\left\langle H_{2}+H_{3}\right\rangle$ for $|1\rangle-\left|K_{2}\right\rangle$. The coefficients $\alpha$ suggest 
two tendencies: holes prefer to sit on the edge of the $4 \times 4$ lattice with many optimal $H_{3,0}$-couplings and holes prefer to sit on the edge of the $\ominus$ squares. The disturbance by holes on the edge of the open-ended lattice of the resonance of short singlet bonds is smaller than by holes in the interior of the lattice. That is why the position on the edge benefits the $H_{2}$-energy.

In the $2 \times 2$ squares denoted by $\theta$, the two-hole configurations with the holes on Manhattan distance one are optimally coupled via $H_{3,1}$. In $\oplus$ the $H_{3,1^{-}}$ couplings are non-optimal.

In the $2 \times 2$ squares of $\left|K_{1}\right\rangle$ and $\left|K_{2}\right\rangle$ - both do not have $\oplus$ or $\Theta$ squares there are as many optimal as non-optimal $H_{3,1}$-couplings. In $|1\rangle$ there are four $\ominus$ squares versus five $\oplus$ squares and in $|2\rangle$ there are five $\ominus$ squares versus four $\oplus$ squares.

With these arguments, onc can understand the order of $\left\langle H_{2}+H_{3}\right\rangle$ for $|1\rangle-$ $\left|K_{2}\right\rangle$. State $|1\rangle$ has $\ominus$ squares lying more in the interior of the lattice, causing a mixture of $H_{3,0-}$ and $H_{3,1}$-phases. States $\left|K_{1}\right\rangle$ and $\left|K_{2}\right\rangle$ do not have the restriction of $\ominus$ and $\oplus$ squares. State $|2\rangle$ has the advantages of both tendencies.

\subsubsection{First-order states}

The influence of $H_{1}$ is visible in the column of table II with label $\left\langle H_{\mathrm{H}-\mathrm{A}}\right\rangle$. As mentioned earlier, the optimal first-order two-hole configurations represent an interaction between the holes. In $\ominus$ squares there are four optimal first-order configurations. In $\oplus$ squares the first-order configurations are not optimal and thus not added to the linear combination approximating the GS. The coefficients $\alpha$ and $\left\langle H_{\mathrm{H}-\mathrm{A}}\right\rangle$ for $|1\rangle-\left|K_{2}\right\rangle$ make clear that the state is more favourable if it has more $\ominus$ squares which are optimally coupled by $H_{3,0}$. The state $|(0,0)\rangle$ has nine $\ominus$ squares, which are optimally coupled by $H_{3,1}$. From table II we conclude that the overall GS for the $4 \times 4$ lattice in this approximation is the bound state $|(0,0)\rangle$.

It is an open question whether the bound state is still the GS if one includes more first-order states. Namely those with the SE not neighbouring both holes or even one of the holes (via $H_{2}$ an SE can move over its sublattice). Furthermore, the calculations show the following tendencies:

- An SE prefers to have as little as possible nearest- and next-nearestneighbour spins. This is due to the $H_{2}$-expectation value.

- For the states $|1\rangle-\left|K_{2}\right\rangle$ the absolute values of the coefficients of the two-hole configurations become smaller if the distance between the holes increases. See fig. 9.

The last tendency is due to two effects. The optimal $H_{1}$-couplings stimulate the holes to be neighbours. Note that the amplitude of $H_{1}(t)$ is larger than the amplitude of $H_{3}\left(t^{2} / U\right)$. The optimal $H_{3,0}$-couplings to the configurations with 


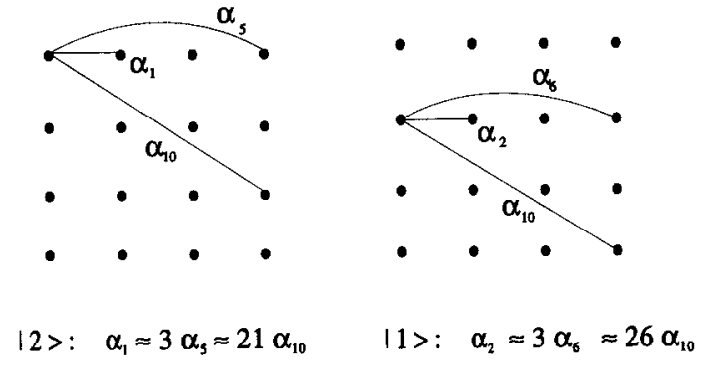

Fig. 9. Coefficients of some two-hole configurations.

two neighbouring holes explains the order of the coefficients $\alpha_{5}$ and $\alpha_{10}$ in $|2\rangle$ and $\alpha_{6}$ and $\alpha_{10}$ in $|1\rangle$. See fig. 9. The question is how far the decrease of the coefficients goes for macroscopical systcms. If $\lim _{r \rightarrow \infty}|\alpha|=c$ with $c>0$, then the best approximation of the GS based on optimal $H_{3,0}$-phases has a large entropy advantage in comparison with the bound state $|(0,0)\rangle$. If $c=0$, then the two holes are bound too, but not as drastically as in $|(0,0)\rangle$.

In view of the size of the calculations, it is necessary to use a different calculation method for larger systems. An alternative may be to derive an effective Hamiltonian, describing the holes and the SE as quasi-particles. Such a Hamiltonian can possibly cope with a larger number of holes and SE. Furthermore, it can be more manageable than the H-A Hamiltonian.

\section{References}

[1] P.W. Anderson, Science 235 (1987) 1196.

[2] C. Gros, R. Joynt and T.M. Rice, Phys. Rev. B 36 (1987) 381.

[3] S. Liang, B. Doucot and P.W. Anderson, Phys. Rev. Lett. 61 (1988) 365.

[4] W. Marshall, Proc. R. Soc. A 232 (1955) 48.

[5] M.R.M.J. Traa and W.J. Caspers, Physica A 183 (1992) 175.

[6] P.L. Iske and W.J. Caspers, Physica A 146 (1987) 151.

[7] M. Tinkham, Group Theory and Quantum Mechanics (McGraw-Hill, New York, 1964) p. 325. 